\title{
Design and Development of Actuating System for Diagnostics Application in Minimally Invasive Surgery (MIS)
}

\author{
Shahram Payandeh, Jason Rothe and Ash Parameswaran \\ Experimental Robotics Laboratoty, School of Engineering Science \\ Simon Fraser University, Burnaby, British Columbia, Canada, V5A 1S6
}

\begin{abstract}
This paper presents some initial concepts in the design and development of actuating systems based on shape memory alloy for Minimally Invasive Surgery (MIS). Specially the motivation is to design tissue/fluid storage mechanism which can be used to collect samples from the surgical area for further diagnostic procedure. The proposed hybrid design combines passive source of energy with the active source. The passive source is based on the storage of spring energy and the active source is based on utilization of shape memory alloy as a part of switching mechanism. Various design concepts for the switching mechanism are presented combined with the design of their prototypes.
\end{abstract}

Keywords - Minimally Invasive Surgery (MIS), tissue/liquid storage mechanisms, hybrid actuating system, shape memory alloy

\section{INTRODUCTION}

Over the last twenty years, minimally invasive surgery (MIS) has become the method of choice for a number of surgical procedures. While open surgery requires the creation of large access wounds in order to expose the relevant anatomy and allow room for manipulation, resulting in a high ratio of access wound trauma to procedure trauma, MIS provides access to the operation site via small wounds, dramatically reducing post-operative pain and shortening recovery times. However, while MIS offers tremendous advantages for the patient, it still suffers from a number of inherent problems that limit its application [1].

Unlike open surgery, MIS requires that all instruments required for the operation pass through a small 5 or $10 \mathrm{~mm}$ access port. This constraint has dramatically affected the nature of instrument design and, to some extent; the future of MIS depends on how well this problem is addressed by the engineering community. The capabilities of MIS can only advance so far without a radical redesign of the devices presently being used. With this motivation in mind, we are looking into ways by which we can develop the next generation of surgical tools.

The specific area of investigation that interests us at this time is that of tissue and fluid sampling and modeling. One of the most significant capabilities offered by MIS techniques is the ability to examine and diagnose possible afflictions without making any significant access wounds. In some cases, diagnosis requires an examination via endoscope or optical catheter while in other cases it also requires a biopsy of specific body tissue or fluid. Tools previously designed to collect these samples during open surgery must now be redesigned to work in a minimally invasive setting. In order to effectively design tools to interact with body fluids and tissues in particular, engineers must have a thorough understanding of the mechanical behavior of these materials. To model this behavior, however, a large amount of data must be collected from living tissues. Providing access to these tissues in situ is another area where MIS becomes very useful, provided the right tools are available. We are therefore investigating the design of tools not only for practicing minimally invasive surgery, but for measuring the mechanical response of living tissues during minimally invasive surgery.

This paper presents some new concepts for designing actuating mechanism, which can be used in the design of fluid sampling and tissue collecting mechanism. The design is based on hybrid actuation concept where both the active and passive sources are combined which can result in an effective approach in MIS application. In the following, first we present some general category of tissue manipulation in MIS, then an overview of actuation concepts and finally various design prototypes that have been developed.

\section{TISSUE MANIPULATION TASKS}

\section{A. Tissue Identification}

During a biopsy tissue sampling procedure, it is often required to remove a sample from a specific organ; therefore, before any sample can be taken the correct organ must be positively identified. This usually requires that the organ be within visual range of the surgeon. It would be useful to have a biopsy sampling tool that can assist the surgeon with the task of identifying the correct tissue either by providing additional visual information (such as by carrying an optical fiber etc.), or perhaps by somehow "pre-sampling" or otherwise testing the tissue by non-visual means. An example would be to use sensors to collect preliminary force/displacement (elasticity) data on the organ in question [2].

\section{B. Tool Guiding and Placement}

Often a very specific piece of tissue, such as a small potentially cancerous area, must be located and sampled. This requires very precise placement of the biopsy tool during the sample excision. The surgeon needs to know that the sample has been taken from the exact area required and not from the surrounding tissue. This can be challenging, 
particularly for organs that are not easily viewable or accessible, or for organs that need to be sampled on their underside or at other difficult locations.

\section{Consistent Tissue Removal Means}

Biopsy tools must also offer reliable operation. Some devices currently in use do not always remove a proper piece of tissue and sometimes do not remove any tissue at all. While it is impossible to achieve $100 \%$ reliability, it is important that a biopsy device be highly effective over as wide an array of tissue types as possible.

\section{Cauterization of the Wound and Sample Damage}

If a larger tissue sample is taken, it is often necessary to cauterize the wound to stop internal bleeding and prevent infection. The preferred method of cauterization during minimally invasive procedures is electrocautery, which has the potential to damage the tissue sample taken if cauterization is performed by the biopsy tool itself [4]. It is preferable to have a biopsy tool that also performs the cauterization since then the biopsy site need only be accessed once using a single tool. With hard to reach biopsy sites in particular, it would be difficult and time-consuming for the surgeon to find the exact place where the tissue was removed with a new set of tools.

\section{E. Tool Size and Sample Size}

All tools designed for MIS must be narrow enough to pass through either a $5 \mathrm{~mm}$ or $10 \mathrm{~mm}$ cannula. What's more, all portions of body tissue to be removed from the body must also pass through this small hole. With the exception of some excisional biopsies, most biopsies require a relatively small piece of tissue to be removed. For situations that require a larger tissue sample, devices have been designed that compress the tissue such that it will conveniently fit through the opening of the cannula and can be expanded again once outside the body cavity.

\section{III.ACTUATION}

It is useful to have a non-rigid version of a biopsy tool that can be used to reach under and around organs or into other difficult areas. The mechanics of such a tool must therefore be mostly confined to the tip of the tool, where flexion does not occur. Actuation then, must either be conveyed through the flexible shaft, or take place at the tip of the tool itself. This section describes our process of developing "proof-of-concept" prototype of a body fluid sampling device designed specifically for MIS. It describes each stage of the design process and the evaluation of our prototypes. It also provides a basic modeling results which can be used in determining some of the design parameters such as spring coefficients.
In the following we assume that the active part of MIS diagnostic tool is located at the tip of the tool. The objective is to design an actuating mechanism that can be used in the design of the fluid sampler for diagnostic purposes[3][6][7].

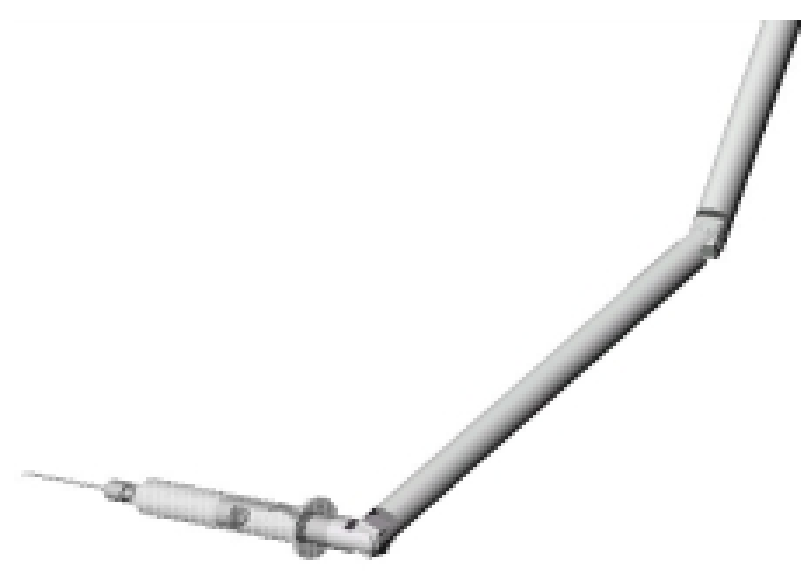

Figure 1. An example of MIS diagnostic tool with active tip.

\section{A. External Mechanically Coupled Actuators}

Almost all commercially available tools for MIS utilize a long, sometimes flexible, coupling to transmit mechanical force/motion from outside the body to the tool within the body. Usually, a stainless steel rod is used to transmit linear and rotational motion generated by the surgeon's hands into the necessary force/motion at the tool. When a more flexible coupling is required, thin wires and/or a series of joints can be used to create the required movements of the tool. The disadvantage of these mechanisms is that they can become complex and bulky when the tool tip is required to have several degrees of freedom.

\section{B. Stored Energy Actuators}

Stored energy actuators are devices such as springs that are "loaded" outside the body cavity and then triggered once inside the body. Their advantage is that they are relatively small and can provide quick, consistent and direct forces/motions at the point of interest. Unfortunately, they can usually only be used once before they must be "reloaded". Also a triggering stimulus must be provided via either an external mechanical coupling or and electrical signal.

\section{Internally Coupled (in vivo) Actuators}

Most internally coupled actuators are electromechanical in nature; that is, they convert energy transmitted from outside the body via an electrical connection into useful mechanical forces/motions within the body. Some examples are small motors, solenoids, piezoelectric devices, and shape memory 
alloys. Actuators that are not electromechanical in nature can also be used. These might include hydraulic and pneumatic devices that convert differences in fluid/air pressure into mechanical forces/motions. Actuators that fit inside the body are advantageous because they can be controlled via very small, flexible connections to the outside world (i.e. wires or small hoses). They do, however, have their drawbacks. Using electricity inside the body is inherently dangerous and is therefore subject to strict regulation. What's more, actuators used within the body must not only be clean, sterile and sometimes biocompatible, but they must be made extremely small. Often, this size restriction means that the performance of actuators used in vivo is limited to small forces/displacements.

\section{DESIGN CONCEPTS}

As a means by which to begin tackling this very large problem of measuring, modeling and sampling living tissues, we have chosen the following approach. While doing preliminary research on tissue sampling and tissue property measurement, we started developing a "stored energy" type of actuator that can be triggered while in the body using only a low-voltage electrical connection. Our intention, in developing this device, is to provide a way to automatically capture tissue/fluid samples from within the body via MIS techniques. By using a stored energy actuator in combination with a small, electromechanical trigger mechanism, we can eliminate the need for complex, bulky and restrictive mechanical linkages to the tool tip. This makes mounting the tool on the end of a flexible stem a very practical possibility. Our first step has been to combine such a trigger mechanism with a fluid sampling device actuated by energy stored in a spring. We began by experimenting with simple miniature fluid pumping mechanisms and eventually ended up developing a miniature electromechanical latch (MEL) capable of releasing a spring-loaded syringe. All the components of the MEL are confined within the diameter of the syringe, which can be mounted on the end of a flexible stem as shown in the Figure 1.

Figure 2 shows an example of concept 1 of electromechanical latch mechanism. The schematics show both release and held position. The design utilizes SMA [8] and simple leaf springs. Two types of SMA with 150 micrometer and 250 micrometer were developed. The figure also shows the developed prototype of this concept. In this design it was observed that the SMA can create small motion and it created force magnitude was not large enough to sufficiently move the springs. Figure 3 shows an example of concept 2 for electromechanical latch mechanism that occupies less space. As shown in the Figure, it consists of a leaf spring, Aluminum and Brass plugs and SMA. The problem with this prototype was that it was difficult to electrically isolate the spring with the aluminum plug. The other problem was due to the preloading of SMA, it does not return to its original configuration when the power is removed.

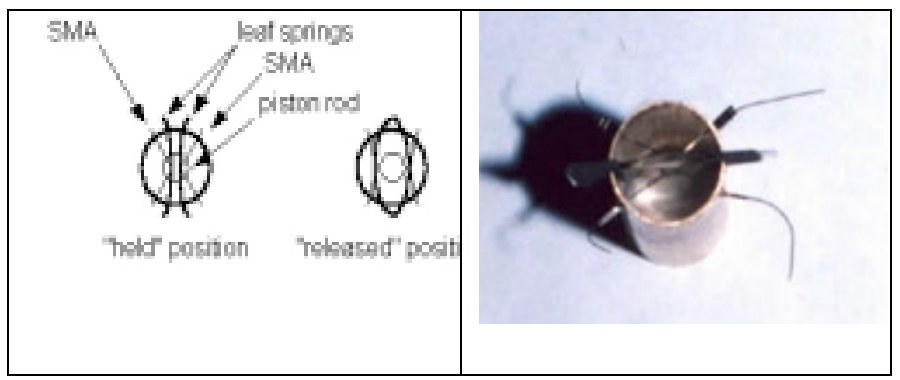

Figure 2. Concept 1 - two central leaf springs/SMA configuration.

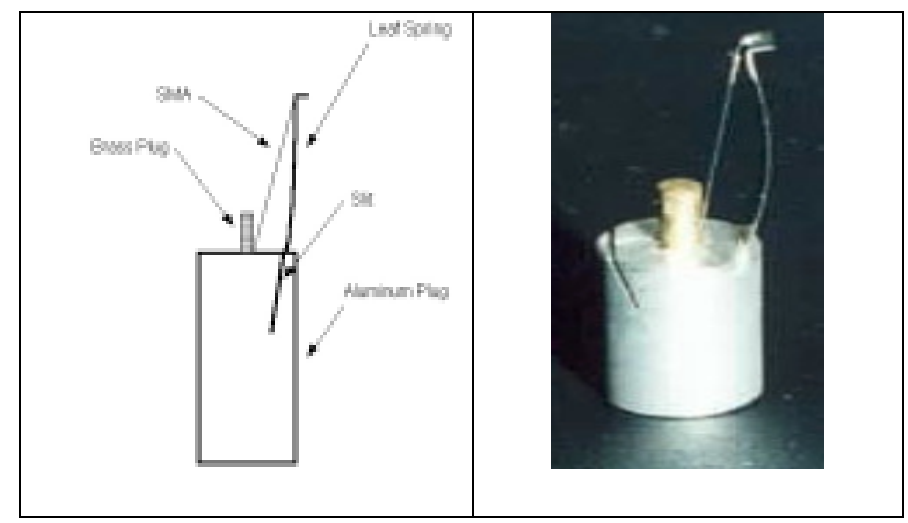

Figure 3. Concept 2 - one leaf spring with single SMA.

Figure 4 shows another concept (Concept 3) which was investigated. This design consists of single leaf spring that catches in a thin groove in a long cylinder. Cylinder has a hole about $5 \mathrm{~mm}$ before the groove to bit a small pin that pushes against the leaf spring to dislodge it from the groove. A SMA wire in a $\mathrm{V}$ configuration actuates the pin. The configuration for the latch mechanism works as expected. However, the disadvantage of the design is that components must be mounted on the outside of the cylinder. Hence, this design cannot be integrated as a latch located at the tip of the sampling device.

Figure 5 shows a design concept (concept 4) where a Derlin plug with two shorter and thicker leaf springs having outward angles were used. The leaf springs have a slot machined in them so that an insulated SMA wire can path through them. This design concept performed the triggering and unlatching actions, however, the design does not create enough preloading of the SMA. This design was further synthesized to result in an optimal design. The complete prototype consisting of the passive spring loaded cylinder and the active latch based on the final design concept is shown in Figure 6. 


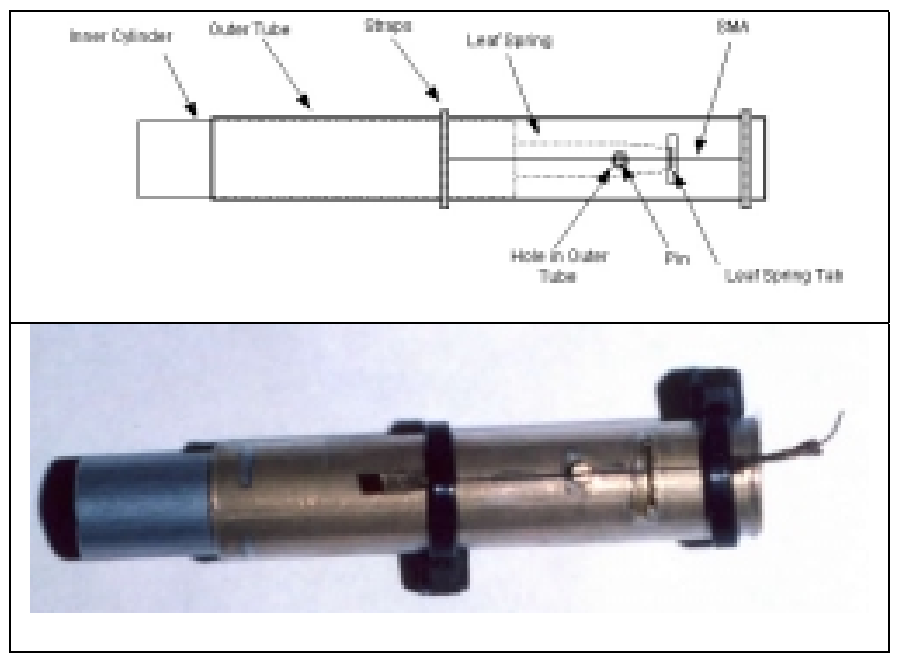

Figure 4. Concept 3- leaf spring with SMA in V configuration.

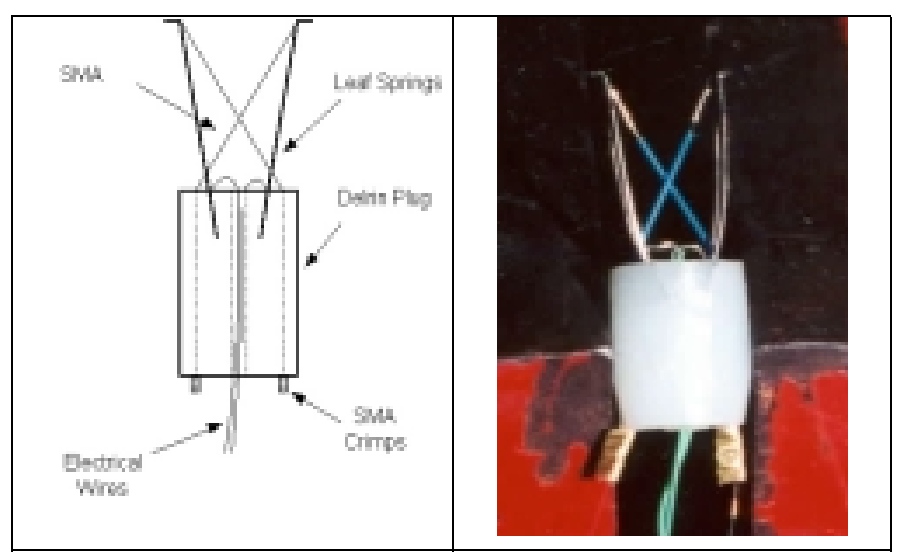

Figure 5. Concept 4 - Two-leaf springs with SMA in $X$ configuration.

\section{DISCUSSIONS}

The ultimate goal of this research is to design a prototype concept similar to the one shown in Figure 1. Combined with the earlier prototypes presented in [3] for design of flexible stem tools for MIS, work is underway to integrate both designs. The major finding of our research is that an optimal design can be a combination of passive source which can give a fast response and increase power to mass ratio (such as a passive coiled spring) and an active element which can be used as a binary triggering mechanism. Such design can be considered as hybrid design where the best elements of two sources of energy can be combined. Such design concepts can be used concurrently in a design of tools such as the ones shown in Figure 1.

The design shown in Figure 6 can be used for collecting tissue or liquid samples from the surgical site. The spring constant of the passive element (the coil spring) can be used as design parameters for determining the amount of in-flow at the tip of the device. For example, for a simplified model of the liquid collecting mechanism, the force required to draw liquid through the smaller tube located at the tip of the sampler can be written as:

$$
F=128 \mu \dot{x} \frac{D^{2}}{4 d^{4}} L \frac{\pi d^{2}}{4}=\left(\frac{8 \pi \mu D^{2} L}{d^{2}}\right) \dot{x}
$$

Where $\mu$ is the fluid viscosity, $D$ is the diameter of the collecting chamber, $L$ is the length of the intake tube, $d$ is the diameter of the intake tube. As it can be seen from this equation, one can size the coil spring constant such that the maximum required force for sampling liquid or tissue can be satisfied.

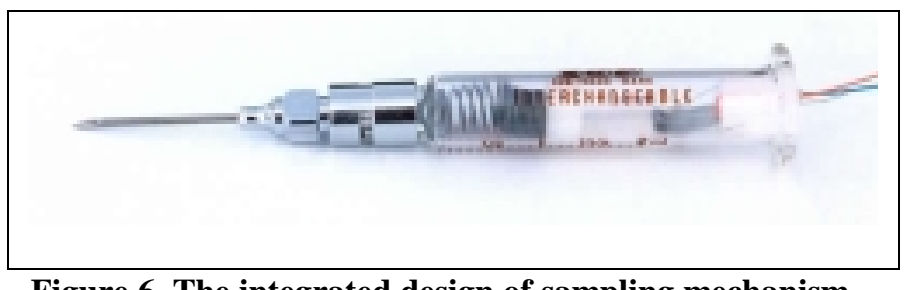

Figure 6. The integrated design of sampling mechanism consisting of passive preloaded coil spring and the latch mechanism based on the Concept 4.

\section{REFERENCES}

[1] A. Faraz and S. Payandeh, "Engineering Approaches to Mechanical and Robotic Design for Minimally Invasive Surgeries (MIS)", Kluwer Academic Publishers, 2000

[2] M. Ottensmeyer and K. Salisbury, Input and Output for Surgical Simulation: Devices to Measure Tissue Properties in vivo and a Haptic Interface for Laparoscopy Simulators, Medicine Meets Virtual Reality 2000, 2000, 236-242.

[3] A. Faraz and S. Payandeh, "Synthesis and Workspace Study of Endoscopic Extenders with Flexible Stem", Trascation of ASME, Journal of Mechanical Design, Vol. 119, Sept. 97, pp. 412-414

[4] G.N.J. Tytgat and J.G. Ignacio, "Technicalities of Endoscopic Biopsy”, Endoscopy 1995; 27: 683-688.

[5] T.G. Frank, G.B. Hanna, A. Cuschieri, "Technological Aspects of Minimal Access Surgery", Proc Instn Mech Eng; Vol 211, Part H, 129-144.

[6] G. Buess, "Update on Clinical Aspects and Technology in Endoscopic Surgery", Endoscopy 1995; 27: 449-454.

[7] K. F. Binmoeller, H. C. Jabusch, H. Seifert, N. Soehendra, "Endosonography-Guided Fine-Needle Biopsy of Indurated Pancreatic Lesions Using an Automated Biopsy Device", Endoscopy 1997; 29: 384-388.

[8] K. Koehn and S. Payandeh, "Evaluation of Shape Memory Alloy Actuators for Minimally Invasive Surgery", Proceedings of 1995 IEEE Systems, Man and Cybernetics conference, pp. 1991-1997 